\title{
In situ X-ray tomography of aqueous foams: Analysis of columnar foam generation
}

\author{
F. García-Moreno ${ }^{\mathrm{a}, \mathrm{b}, *}$, P. H. Kamm ${ }^{\mathrm{a}}$, T. Neu ${ }^{\mathrm{a}}$, K. Heim ${ }^{\mathrm{b}}$, A. Rack ${ }^{\mathrm{c}}$, J. Banhart ${ }^{\mathrm{a}, \mathrm{b}}$ \\ a Applied Materials, Helmholtz-Zentrum Berlin für Materialien und Energie, Hahn-Meitner-Platz 1, 14109 Berlin, Germany \\ b Institute for Materials Science and Technology, Technische Universität Berlin, Hardenbergstrasse 36, 10623 Berlin, \\ Germany \\ ${ }^{\mathrm{c}}$ European Synchrotron Radiation Facility, 71 Avenue des Martyrs, 38043 Grenoble, France \\ * Corresponding author, email: garcia-moreno@helmholtz-berlin.de, Tel. +49 30 806242761, Fax: + 4930806243059
}

\begin{abstract}
The accumulation of bubbles rising in a liquid and forming a foam is a complex process which is difficult to observe and describe in three dimensions due to the poor visibility of bubbles in the depth of a foam when using optical microscopes. We applied in situ X-ray tomography in phase-contrast mode to visualise how bubbles of almost equal size accumulate and form a foam confined within a vertical tube in an aqueous liquid. By capturing a full 3D image of the foam after the arrival of each bubble and tracking each bubble individually, collective movements of bubbles, displacements and changes of positions could be described unambiguously.
\end{abstract}

Graphical abstract:

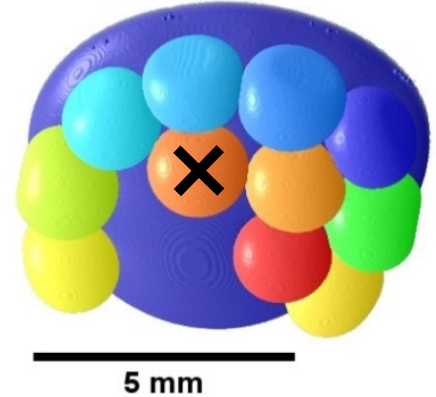

11 bubbles

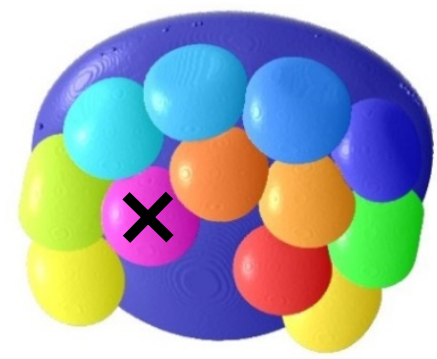

12 bubbles

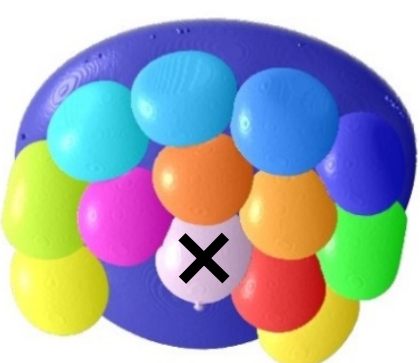

13 bubbles

$3 \mathrm{D}$ rendering of a sequence of tomographies of an aqueous foam made by adding one bubble every minute to a liquid/gas interface (big blue surface). By analysing the images one can follow the build-up of the foam structure in detail. Crosses mark the bubbles that have arrived last in each image. 
To study bubble arrangements in foams in three dimensions, to measure the local liquid fraction within, order parameter of foams or other properties, X-ray tomography is a suitable and accepted method [1-3]. Recent developments at synchrotron X-ray tomography beamlines have led to such high tomography acquisition rates that liquid foams can now be studied in situ and dynamic processes such as bubble movements, coarsening or drainage observed in the entire foam volume with high time resolution. [4-11] Such time-resolved tomography is also called '4D tomography'.

The arrangement of monodisperse aqueous bubbles produced by gas injection into a liquid is of special interest [12-16]. Optical observation allows only for the study of the structure of the first three flat surface layers in crystalised monodisperse foams [13, 17], but can hardly reproduce the inner structure of a bulk foam as X-ray tomography permits [18]. Light scattering experiments probe a deeper volume but only average information about bubble sizes in the bulk is provided and other limitations apply [3, 19-21]. Simulations based on packings of hard spheres have been used to understand the structure of monodisperse bubbles in cylindrical columns [22-25], and recently also combined with experiments [26]. But such simulations are restricted to ratios of the cylinder diameter $D$ to the bubble diameter $d$ of $D / d<2.9$ [22] and recently until $D / d=4$ [25] as computation time increases exponentially with increasing ratio. Tomographic images of monodisperse aqueous foams constrained in columns were recorded and helped to illustrate arrangements of real bubble in such systems [27]. Such experiments open access to larger $D / d$ ratios and provide the positions of all bubbles inside a specimen. The structures found differ from ideal structures due to several reasons: i) a slight deviation from monodispersity of bubble sizes, ii) the deformation of the bubbles compared to hard spheres, iii) an alignment of the bubbles along a curved instead of a flat surface due to the meniscus shape of the liquid/gas interface, and iiii) defects and rearrangements created by the interaction between resting and injected bubbles, i.e. by the impact of incoming bubbles accelerated by buoyancy forces [28].

In the work presented here, the generation of a foam column was studied by observing the attachment of individual bubbles to a liquid gas interface in situ using X-ray tomography. Such in situ 4D tomography with moderate time resolution has been used to research dynamic effects such as coarsening [3] or crystallisation [29] and was used in the present work, with increased time resolution, to study the build-up of a foam constrained in a cylindrical tube.

\section{Experimental}

\subsection{In situ generation of individual bubbles}

Bubbles were produced by injecting air through a stainless steel cannula of $200 \mu \mathrm{m}$ inner diameter into a vertically arranged thin-walled tube of diameter $D \approx 8.5 \mathrm{~mm}$ filled with an aqueous solution (Fig. 1). The tube was made of polyimide that transmits X-rays very well. To obtain stable foam we used an aqueous solution containing $5 \mathrm{vol} \%$ of the commercial detergent Fairy Liquid and to reduce the coarsening rate we enriched the blowing air with perflourohexane vapor that is insoluble in the liquid [30]. Individual bubbles of a controlled size were generated by defining pressure pulses using a very fast magnetic valve (type MHE2-MS1H-3/2G-M7-K from Festo, Germany) for the opening and closing of the gas supply during a period of time as short as $1.7 \mathrm{~ms}$. The valve was controlled by selfdeveloped hardware, which allows for adjusting the opening time and the interval between the pulses independently and also to generate single pulses. Adjustment of the opening time on the one hand and the gas pressure in the line with a needle valve on the other allowed us to 
control the gas volume of each pulse. Single bubbles of a similar pre-set diameter $d$ ranging from $1.5 \mathrm{~mm}$ to $5 \mathrm{~mm}$ could be produced. The pre-set gas volume $V \approx 3 \mathrm{~mm}^{3}$ used in the experiment corresponded to a spherical bubble diameter of $d \approx 1.7 \mathrm{~mm}$, i.e. to a nominal cylinder-to-bubble diameter ratio $\lambda=D / d \approx 5$, going beyond the simulation limits [25]. For our experiment, we chose an interval between gas valve activation of one minute, i.e. produced one individual bubble every minute. This time interval allowed us to record a complete tomography in $30 \mathrm{~s}$, inject the next bubble and let the foam column relax for a few seconds, during which the gas-liquid interface that had been slightly pushed up during gas injection by the liquid in the line returned to its original position, before recording the next tomography. In total 48 bubbles were produced in the tube, after which the experiment was terminated.

\subsection{In situ 4D tomography}

To allow for 4D tomography, the whole gas injection setup including the cylindrical tube containing the aqueous solution, a washing bottle containing perflourohexane, the injector cannula, the magnet valve and a source of pressurised air (a small electrical air pump) was fixed on a rotation stage and rotated continuously (Fig. 1). Several electrical sliding contacts allowed us to connect the devices on the rotating table to the external hardware without limiting the maximal amount of continuous rotations.

The experiment was carried out at the imaging beam line ID 19 of the European Synchrotron Radiation Facility (ESRF), Grenoble, France. The beam line provides different polychromatic configurations with high photon flux density in the energy range of 18-250 keV. The radiation transmitted through the foam was transformed to visible light by a 100- $\mu$ m thick LuAG:Ce scintillator, after which the image was guided to the camera sensor using a mirror and lenses. A pco.dimax high-speed camera from PCO AG, Kelheim, Germany, with $2016 \times 2016$ pixels together with an optical magnification of 2 allowed us to obtain a field of view of $(10.2 \times 10.2) \mathrm{mm}^{2}$ with each pixel representing an area of $5.06 \mu \mathrm{m}$ edge length in the sample. Although faster image acquisition rates than the one chosen would have been feasible, we selected a polychromatic beam with narrow bandwidth of around $2 \%$ (undulator U17-6C, gap $29.5 \mathrm{~mm}$ ) with an energy of $19 \mathrm{keV}$ and a limited flux to prevent radiation damages of the foam sample. In particular, we recorded a tomography in $30 \mathrm{~s}$ (300 projections $/ 1^{\circ}$ ) with $35 \mathrm{~ms}$ exposure time per projection (Fig. 2). A drift space between sample and detector allowed for the application of propagation-based phase contrast in order to visualise the weakly attenuating cell walls.

4D tomography does not only visualise the foam structure and its evolution from one bubble injection to the other, but also allows us to quantify bubble positions, bubble volumes, local or integral liquid fractions, foam inner surfaces, number of neighbours, order parameters, etc. [18]. As in our experiment bubble sizes were in the mm range, they could be deformed and then deviated from the ideal spherical shape depending on their position and the number of their neighbours. Therefore, for each bubble we defined its bubble diameter as the equivalent diameter of a sphere of the same volume as the bubble extracted from the tomographic data set.

As shown in Fig 3, individual bubbles could be separated and labelled with different numbers or colours. As the gas volume of each bubble could be measured very precisely, individual bubble tracking due to the slightly different bubble volumes was possible, e.g. allowing us to monitor whether a single bubble was displaced or exchanged its position with 
another bubble or whether a whole cluster of bubbles was rotated under the influence of the incoming bubble.

\section{$3 \quad$ Results and discussion}

A total number of 48 bubbles were injected and corresponding tomographies acquired after each injection. Bubble rearrangements of different magnitude, bubble displacements within a bubble layer as well as rotations of bubble chains and clusters during the filling of the cylinder were observed. Furthermore, the bubble sphericity and diameter evolution of the bubbles was studied.

\subsection{Bubble chain and cluster rotation}

To illustrate better the bubble by bubble and layer by layer growth of the columnar foam we virtually rotated the 3D reconstruction and observed the cylinder from the bottom as shown in Fig. 3. Here we see that the preferred position of the first bubbles is the outer part of the column directly next to the wall of the tube. This is understandable as buoyancy pushes the gas bubbles to the top and into the meniscus of the aqueous solution at the air/liquid interface, the highest point of which is at the wall of the polyimide tube where the lowest potential energy is found. This outer bubble chain is shown in Fig. 3. It was built up from initially 3 to 6 bubbles that also rotated clockwise in the course of further bubble injection. Bubbles were pushed away by newly incoming ones, which follow the same path as the previous ones and hit a slightly off-centre position most likely due to the not exactly centred position of the injector cannula. Newly arriving bubbles displaced resting bubbles in the chain due to their higher kinetic energy, which resulted in rotation as the chain does not seem to adhere to a particular point of the tube wall.

Bubble 4 is an exception since it has not moved to the wall of the column but to a more inward position in the second row after injection. This bubble seemingly changed its position relative to the neighbouring bubbles whenever a new bubble arrived and moved from the gap between number 1 and 2 to between 2 and 3, 3 and 5, and so on. However, as the outer chain rotated and bubble 4 moved counter-clockwise in each step, in effect it remained at the same position with respect to the frame defined by the column. Possibly it was pinned to the air/liquid interface by some surfactants or impurities.

Even a complete bubble cluster in the first bubble layer composed of outer but also inner bubbles rotated as shown in Fig. 4. In this case, the rotation was caused by the arrival of bubble number 14. This large structural change led to further bubble rearrangements as shown for bubbles number 11 and 13. Bubble number 14 was deforming the meniscus pulling bubble number 11 out of its place which was afterwards occupied by bubble number 13 .

\subsection{Bubble displacements and ejections}

Fig. 5 shows the displacement of bubble number 16 by the incoming number 17, after which the first bubble layer was completed. Our selection $\lambda=5$ led to a first layer (of a later cylindrical crystal) with positions of the outer (10 bubbles), middle (6 bubbles) and inner bubble ring (1 bubble) as measured previously in an experiment based on microbubbles [27].

A further effect of mechanical instability similar as predicted in the literature could be observed in Fig. 6, namely a bubble (number 19) standing alone in the $2^{\text {nd }}$ layer was displaced by incoming bubble number 20 [28]. Unfortunately, the limited total number of bubbles and layers as well as the low $\lambda$ of our experiment does not allow at this point to distinguish between bubbles that formed a fcc or hcp lattice. One would have to produce inverted bubble 
pyramids and let bubbles impact on their tip to study this point in detail as proposed in Ref. [28]. This could be part of a future experiment.

\subsection{Cylindrical foam packing structure}

Looking at the crystal structure of the final foam (Fig. 7), the helical structure formed by the outer rings is obvious [22, 25, 27]. The outer rings appear to be ordered in an fcc or hcp structure, but as the bubble layer next to the meniscus is shifted to a higher $\mathrm{z}$ position, it is not exactly in a plane, which makes it difficult to make definite statements and comparisons to simulated structures. This problem may be solved by generating more than 3 layers in future experiments. For columnar crystals, structural changes in the packing were reported in the literature [27]. Such changes were explained by the changing liquid fraction over the foam height.

\subsection{Evolution of bubble diameter/volume}

The evolution of the bubble volume over time $V(n)$ was extracted from the tomographies for each bubble $n$ and is displayed in Fig. 8. Not all bubbles had the same volume, but the dispersity of the diameters was just $4.3 \%$ at the end of the experiment. This is less than the threshold of $5 \%$, below which foams can be considered monodisperse [16, 27]. Such small differences in gas volume might have been caused by trigger delays caused by the sliding contacts connecting the rotating valve and the stationary hardware. The mean bubble volume after the arrival of the last bubble was $\bar{V}$ (bubble 48 ) $=3.62 \pm 0.48 \mathrm{~mm}^{3}$, corresponding to a mean diameter of $\bar{d}$ (bubble 48$)=1.90 \pm 0.08 \mathrm{~mm}$.

The gas volume increase of the bubbles corresponds to a volume increase rate of $0.021 \mathrm{~mm}^{3} / \mathrm{min}$ and a total relative increase of $20.6 \%$. All bubbles showed a similar rate with a tendency to a slightly lower rate for higher bubble numbers (the bubbles generated later). As there was no other active gas source, the volume expansion could have been the consequence of the nucleation of gas previously dissolved in the liquid, perhaps due to the continuous X-ray irradiation, or due to a temperature increase in the system. A volume increase of $20.6 \%$ would correspond, following the ideal gas law, to an increase in temperature of $63 \mathrm{~K}$, leading to a final temperature of $\sim 83^{\circ} \mathrm{C}$ if thermal expansion were the only reason.

\subsection{Bubble sphericity}

Another criterion for bubble crystallisation in addition to monodispersity is bubble sphericity $S$ which describes the deviation from spherical shape [31]:

$$
S=\frac{\pi^{\frac{1}{3}}(6 V)^{\frac{2}{3}}}{A}
$$

with $A$ the surface of the bubble. Bubbles tend to be more spherical if they are small. In our case, the average sphericity $\bar{S}$ of the first 17 bubbles in the first top layer (layer 1) was $\bar{S}(1-17)=0.99 \pm 0.01$ after $17 \mathrm{~min}$., which is very close to a sphere, but decreased to $\bar{S}(1-17)$ $=0.96 \pm 0.02$ after $48 \mathrm{~min}$. due to liquid drainage, that makes a foam dryer and bubbles more polyhedral, and the additional buoyancy force acting on the deeper lying layers. This implies that the average sphericity of the bubbles in a layer decreases the more layers are beneath it, which is more likely with increasing foam height. Moreover, the bubbles in the upper layer transformed into a more polyhedral shape with elapsing time, see Fig. 7, as can be expected as the foam dries out due to liquid drainage. If we compare all three layers right after they form (only 14 bubbles for the bottommost layer) then their sphericity is very similar, 
corresponding to $\bar{S}(1-17)=0.99 \pm 0.01$ in layer $1, \bar{S}(18-34)=0.98 \pm 0.01$ in layer 2 and $\bar{S}(34-48)=0.99 \pm 0.01$ in layer 3 (not complete).

\section{Conclusions}

In situ single bubble injection combined with X-ray 4D tomography was performed for the first time, allowing us to study the structure formation and evolution of cylindrical foam columns by quantitative image analysis. We conclude:

- Bubble rearrangements and displacements within layers, ejections out of a layer as well as bubble chain and cluster rotation at the meniscus took place.

- Fcc or hcp ordering of bubbles at the outer layer was observed.

- The volume of the bubbles increased in time, possibly due to a temperature increase caused by X-ray irradiation.

- The sphericity of bubble layers decreased with time with increasing bubble number, i.e. with increasing foam height and liquid drainage.

\section{Acknowledgements}

Funding by ESA (project AO-99-075) and DFG (project BA 1170/35-1 and GA 1304/5-1) are gratefully acknowledged. Experiments were performed on beamline ID19 at ESRF in the framework of the Long Term Proposal MA-1876. 


\section{References}

[1] A. Turbin-Orger, P. Babin, E. Boller, L. Chaunier, H. Chiron, G. Della Valle, R. Dendievel, A.L. Reguerre, L. Salvo, Growth and setting of gas bubbles in a viscoelastic matrix imaged by X-ray microtomography: the evolution of cellular structures in fermenting wheat flour dough, Soft Matter, 11 (2015) 3373-3384.

[2] J. Lambert, I. Cantat, R. Delannay, A. Renault, F. Graner, J.A. Glazier, I. Veretennikov, P. Cloetens, Extraction of relevant physical parameters from 3D images of foams obtained by X-ray tomography, Colloids Surf., A 263 (2005) 295-302.

[3] A. Stocco, F. Garcia-Moreno, I. Manke, J. Banhart, D. Langevin, Particle-stabilised foams: structure and aging, Soft Matter, 7 (2011) 631-637.

[4] A. Rack, F. Garcia-Moreno, C. Schmitt, O. Betz, A. Cecilia, A. Ershov, T. Rack, J. Banhart, S. Zabler, On the possibilities of hard X-ray imaging with high spatio-temporal resolution using polychromatic synchrotron radiation, J. Xray Sci. Technol., 18 (2010) 429441.

[5] H. Takano, K. Yoshida, T. Tsuji, T. Koyama, Y. Tsusaka, Y. Kagoshima, Fast X-ray micro-CT for real-time 4D observation, J. Phys. Conf. Ser. , 186 (2009) 012049.

[6] A. Momose, W. Yashiro, S. Harasse, H. Kuwabara, Four-dimensional X-ray phase tomography with Talbot interferometry and white synchrotron radiation: dynamic observation of a living worm, Opt. Express 19 (2011) 8423-8432.

[7] S. Deville, J. Adrien, E. Maire, M. Scheel, M. Di Michiel, Time-lapse, three-dimensional in situ imaging of ice crystal growth in a colloidal silica suspension, Acta Mater. , 61 (2013) 2077-2086.

[8] H. Takano, M. Morikawa, S. Konishi, H. Azuma, S. Shimomura, Y. Tsusaka, S. Nakano, N. Kosaka, K. Yamamoto, Y. Kagoshima, Development of real-time x-ray microtomography system, J. Phys. Conf. Ser. , 463 (2013) 012025.

[9] R. Mokso, D.A. Schwyn, S.M. Walker, M. Doube, M. Wicklein, T. Müller, M. Stampanoni, G.K. Taylor, H.G. Krapp, Four-dimensional in vivo X-ray microscopy with projection-guided gating, Sci. Rep., 5 (2015) 8727.

[10] E. Maire, C. Le Bourlot, J. Adrien, A. Mortensen, R. Mokso, 20 Hz X-ray tomography during an in situ tensile test, Int. J. Fract. , 200 (2016) 3-12.

[11] P.H. Kamm, F. García-Moreno, T.R. Neu, K. Heim, R. Mokso, J. Banhart, Fast Synchrotron X-Ray Tomography of Dynamic Processes in Liquid Aluminium Alloy Foam, Adv. Eng. Mater. , (2016). 
[12] W. Drenckhan, S. Hutzler, Structure and energy of liquid foams, Adv. Colloid Interface Sci. , 224 (2015) 1-16.

[13] A. van der Net, W. Drenckhan, D. Weaire, S. Hutzler, The crystal structure of bubbles in the wet foam limit, Soft Matter, 2 (2006) 129-134.

[14] W. Drenckhan, D. Langevin, Monodisperse foams in one to three dimensions, Current Opinion in Colloid and Interface Science, 15 (2010) 341-358.

[15] A.J. Meagher, D. McAteer, S. Hutzler, D. Weaire, Building the pyramids: perfect bubble crystals, Philos. Mag. , 93 (2013) 4138-4150.

[16] R. Höhler, Y. Yip Cheung Sang, E. Lorenceau, S. Cohen-Addad, Osmotic Pressure and Structures of Monodisperse Ordered Foam, Langmuir, 24 (2008) 418-425.

[17] A. van der Net, L. Blondel, A. Saugey, W. Drenckhan, Simulating and interpretating images of foams with computational ray-tracing techniques, Colloids Surf., A 309 (2007) 159-176.

[18] A.J. Meagher, M. Mukherjee, D. Weaire, S. Hutzler, J. Banhart, F. Garcia-Moreno, Analysis of the internal structure of monodisperse liquid foams by X-ray tomography, Soft Matter, 7 (2011) 9881-9885.

[19] R. Höhler, S. Cohen-Addad, D.J. Durian, Multiple light scattering as a probe of foams and emulsions, Curr. Opin. Colloid Interface Sci., 19 (2014) 242-252.

[20] D.J. Durian, D.A. Weitz, D.J. Pine, Multiple Light-Scattering Probes of Foam Structure and Dynamics, Science, 252 (1991) 686-688.

[21] M.U. Vera, A. Saint-Jalmes, D.J. Durian, Scattering optics of foam, Appl. Opt. , 40 (2001) 4210-4214.

[22] A. Mughal, H. Chan, D. Weaire, S. Hutzler, Dense packings of spheres in cylinders: Simulations, Phys. Rev. E, 85 (2012).

[23] A. Mughal, H. Chan, D. Weaire, Phyllotactic Description of Hard Sphere Packing in Cylindrical Channels, Phys. Rev. Lett. , 106 (2011).

[24] S.T. Tobin, J.D. Barry, A.J. Meagher, B. Bulfin, C.E. O’Rathaille, S. Hutzler, Ordered polyhedral foams in tubes with circular, triangular and square cross-section, Colloids Surf., A 382 (2011) 24-31.

[25] L. Fu, W. Steinhardt, H. Zhao, J.E.S. Socolar, P. Charbonneau, Hard sphere packings within cylinders, Soft Matter, 12 (2016) 2505-2514.

[26] L. Fu, C. Bian, C.W. Shields, IV, D.F. Cruz, G.P. López, P. Charbonneau, Assembly of hard spheres in a cylinder: a computational and experimental study, Soft Condensed Matter (2016) 11. 
[27] A.J. Meagher, F. García-Moreno, J. Banhart, A. Mughal, S. Hutzler, An experimental study of columnar crystals using monodisperse microbubbles, Colloids Surf., A 473 (2015) 55-59.

[28] S. Heitkam, W. Drenckhan, J. Fröhlich, Packing Spheres Tightly: Influence of Mechanical Stability on Close-Packed Sphere Structures, Phys. Rev. Lett. , 108 (2012) 148302.

[29] A.J. Meagher, D. Whyte, J. Banhart, S. Hutzler, D. Weaire, F. Garcia-Moreno, Slow crystallisation of a monodisperse foam stabilised against coarsening, Soft Matter, 11 (2015) 4710-4716.

[30] D. Weaire, V. Pageron, Frustrated froth: Evolution of foam inhibited by an insoluble gaseous component, Philos. Mag. Lett. , 62 (1990) 417-421.

[31] H. Wadell, Sphericity and Roundness of Rock Particles, J. Geol. Discipline, 41 (1933) 310-331. 


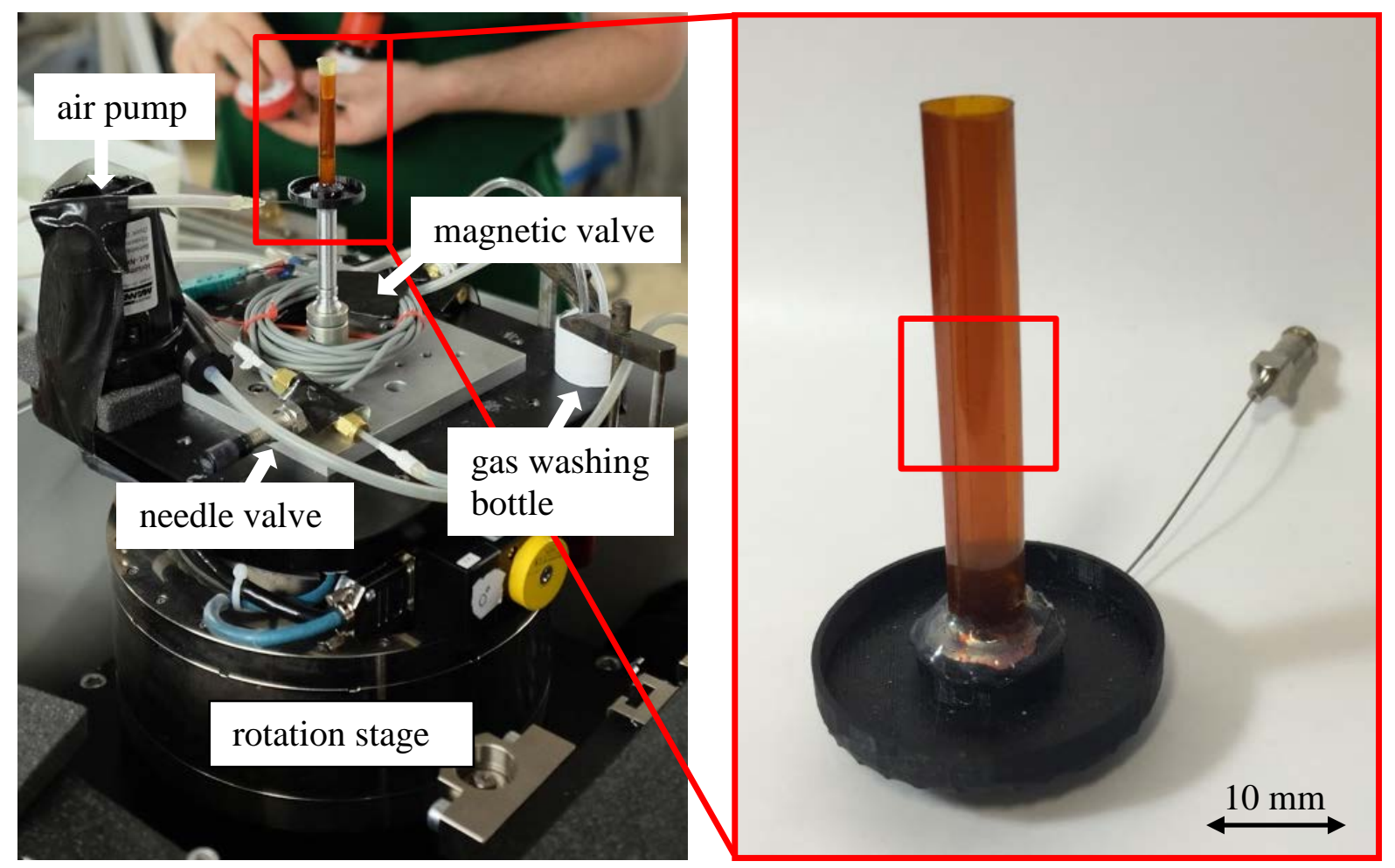

Figure 1. Setup for the in situ observation of bubble and foam generation mounted on a rotation stage and comprising a polyimide cylinder, a stainless steel cannula, an air pump, a gas washing bottle, a needle valve and a magnetic valve. The inset shows a detailed view of the polyimide cylinder with injection cannula and the red square marks the approximate position of region studied, i.e the field of view of tomography. 
a)

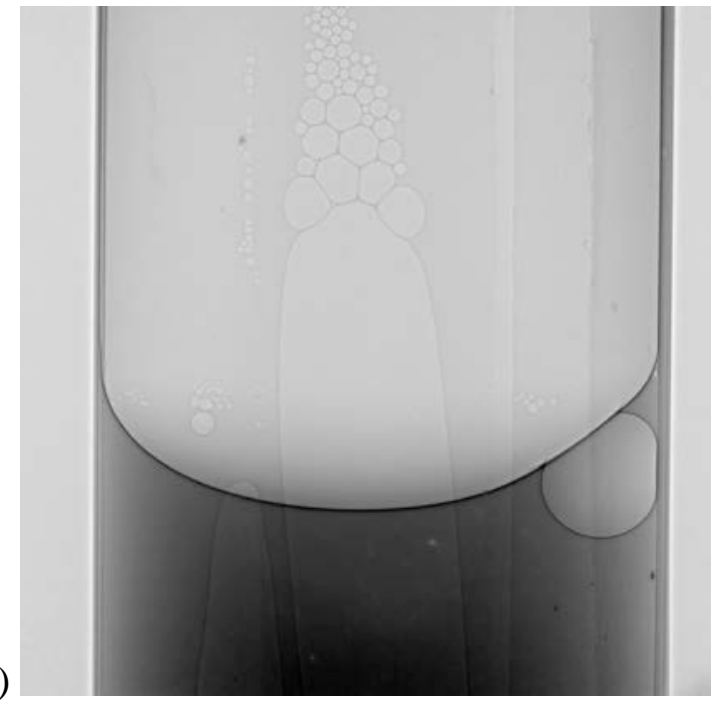

b)

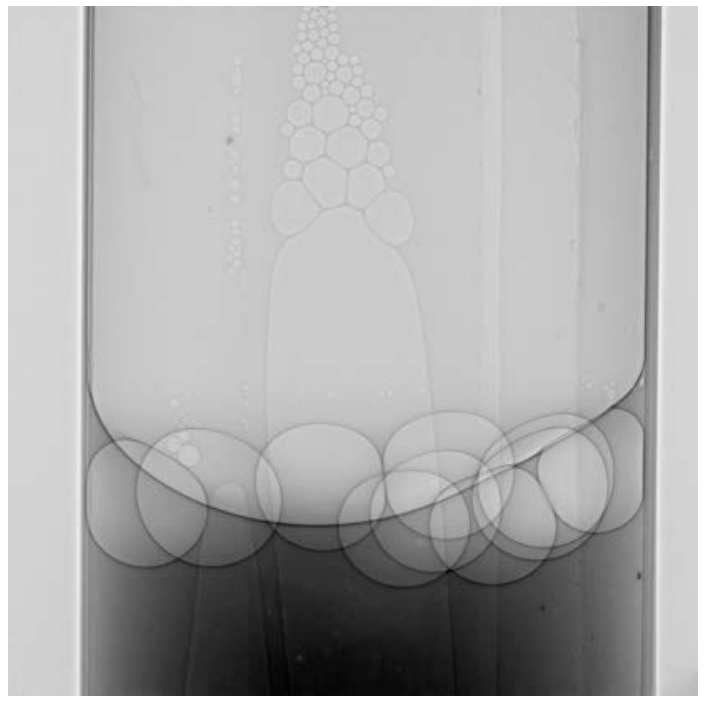

C)

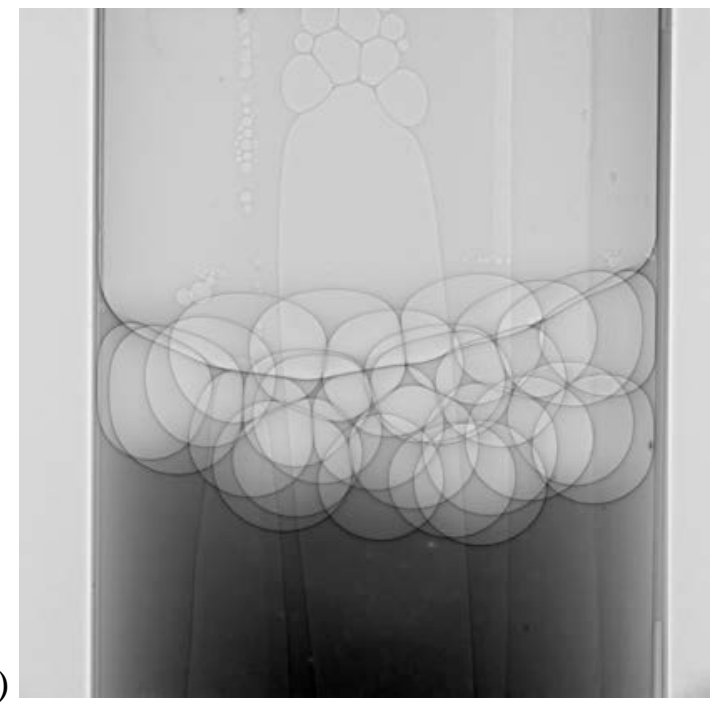

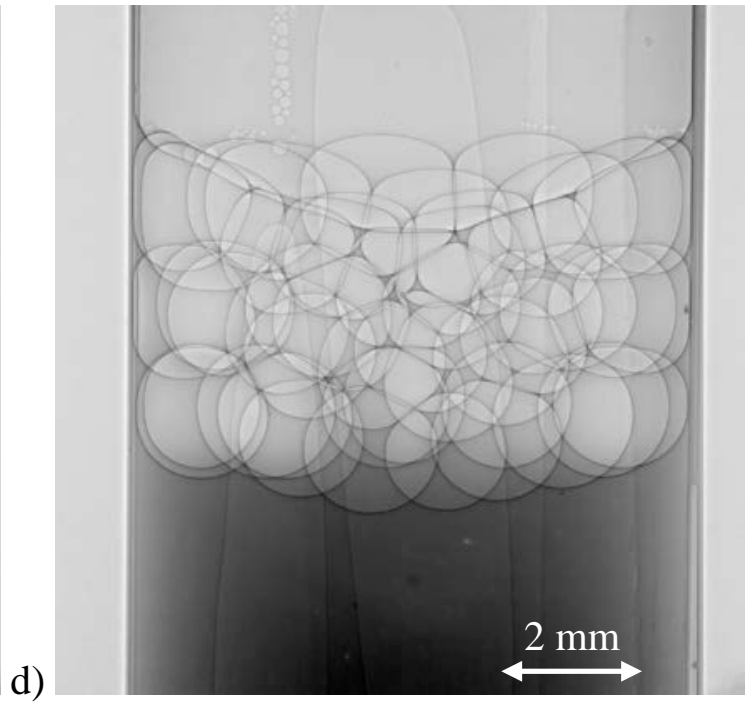

Figure 2. Series of radiographies of the bubbles inside the tube showing the curvature of the meniscus at the air/liquid interface. Images were taken at a) $t=1 \mathrm{~min}, \mathrm{~b}) 10 \mathrm{~min}, \mathrm{c}) 26 \mathrm{~min}$ and d) $48 \mathrm{~min}$ after the start of the experiment (as 1 bubble per minute is injected the number of bubbles corresponds to the number of minutes). Note that these radiographies taken at one specific angle are part of the set of tomographic projections. The small features in the background are small bubbles created when the liquid was poured into the tube and are not relevant here. 


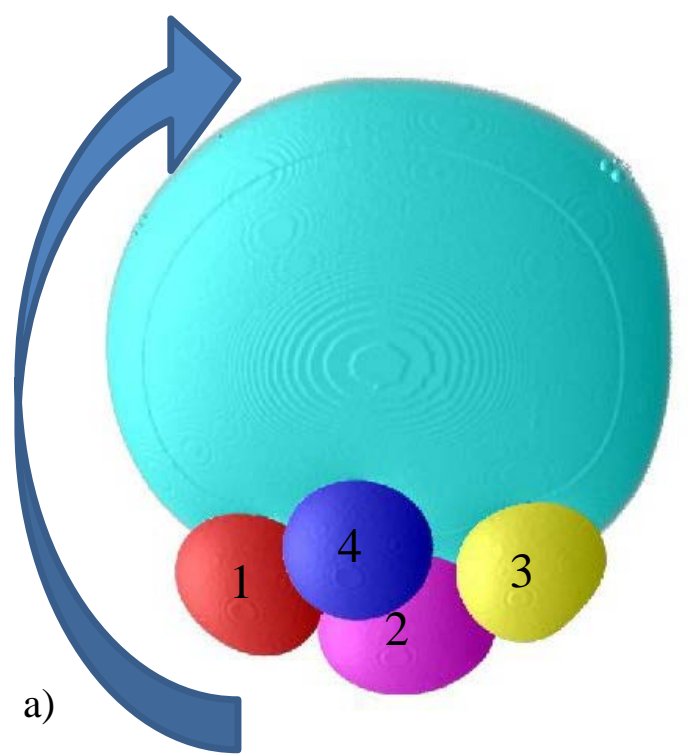

b)
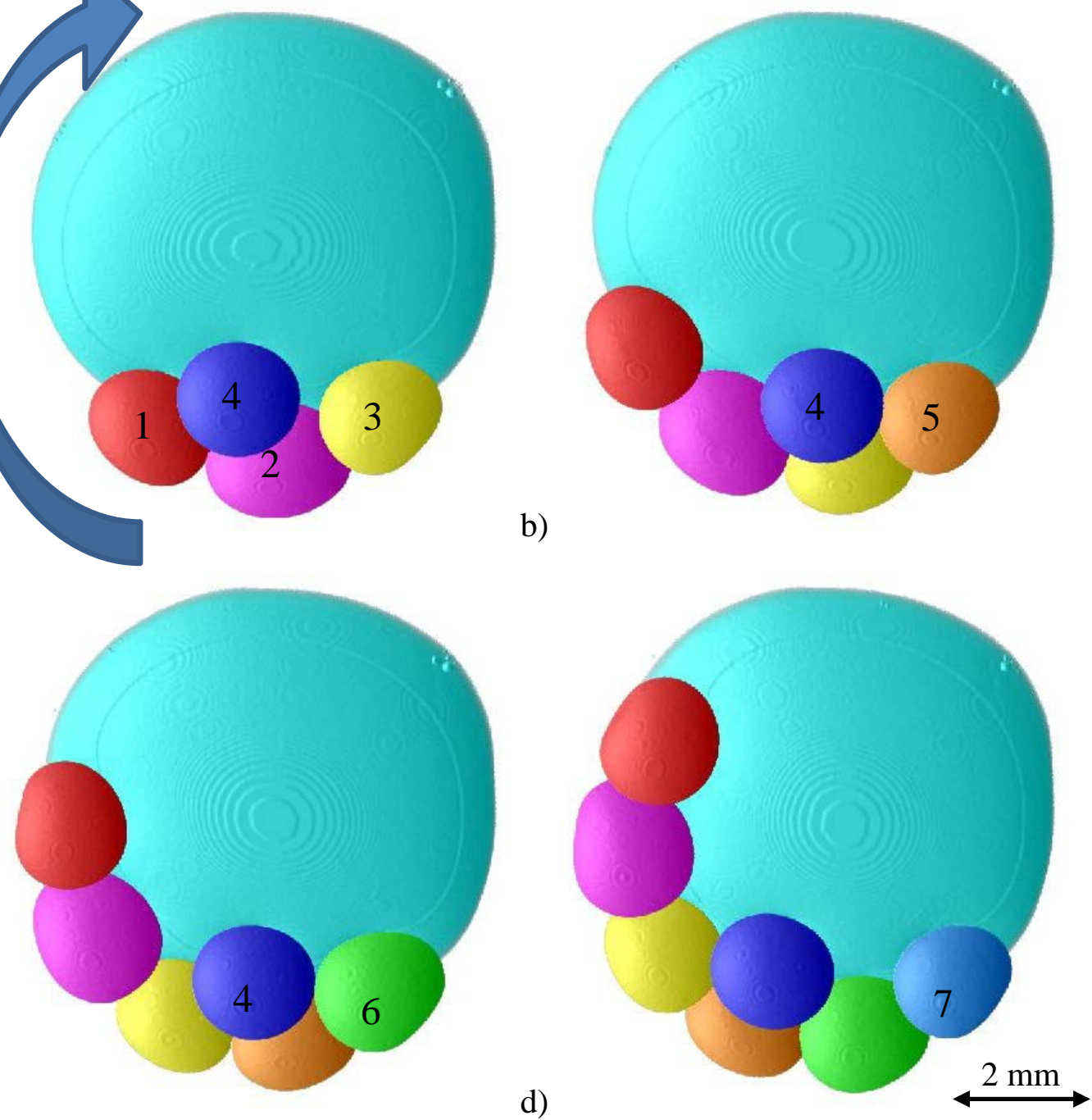

Figure 3. Sequence of tomographies after a) 4 min., b) 5 min., c) 6 min. and d) 7 min. The direction of view is along the column axis from the bottom. Individual bubbles are numbered and colour-coded. A bubble chain lining the wall of the column was continuously built up and increased in length by one for each new bubble floating from the injector towards the bottom of the liquid/gas interface (shown as a large light blue surface). The entire chain was rotated clockwise as new bubbles arrived. Between 4 min. and 5 min., bubble number 4 , which is in a more inward position than the other bubbles, changed its apparent position from the gap between number 1 and 2 to between 2 and 3 and during the ensuing steps the changes continued, but relatively to the tube wall it remained at the same position. 
a)

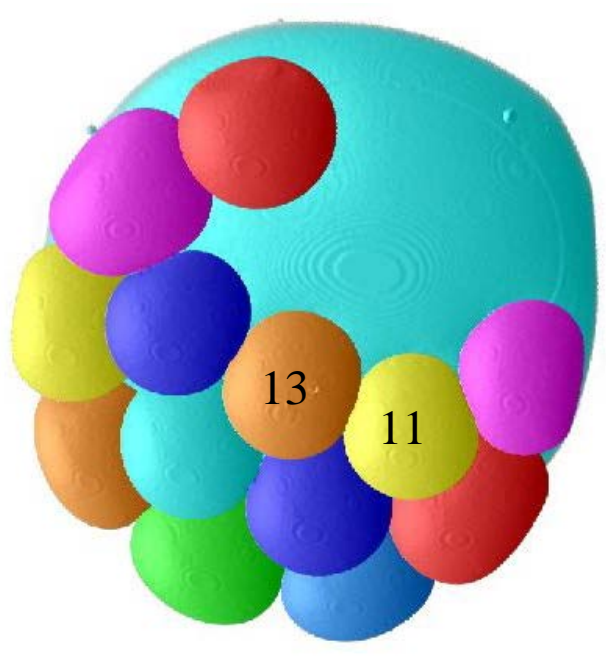

b)

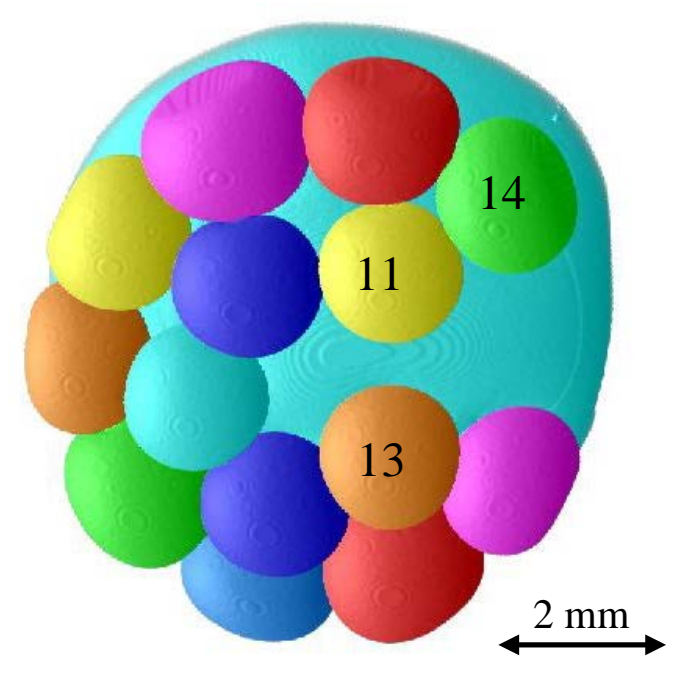

Figure 4. Bubbles displayed as in Fig. 3 but for a later stage: a) 13 min, b) 14 min. The collective rotation of the bubble chain lining the column wall ( 8 bubbles) and 3 bubbles in the second row is observed upon arrival of bubble number 14. Simultaneously a rearrangement of bubbles number 11 and 13 took place. Bubble number 13 took the position of 11 and left a vacancy behind, while number 11 shifted to a new position. 
a)

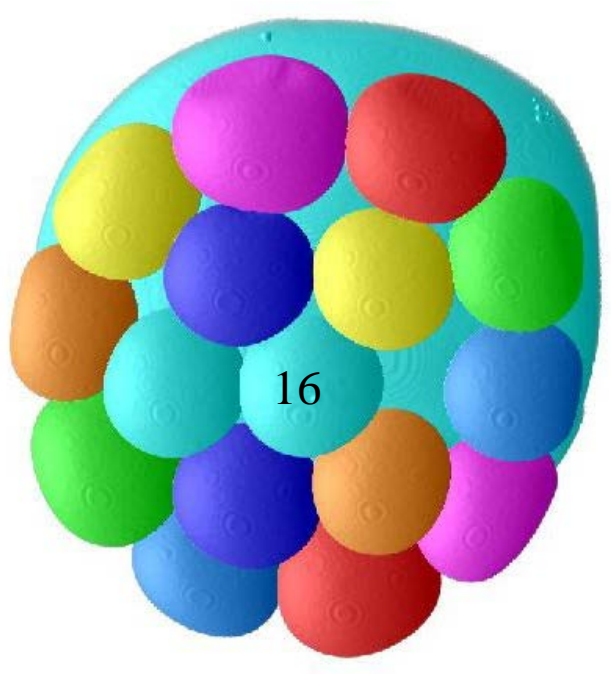

b)

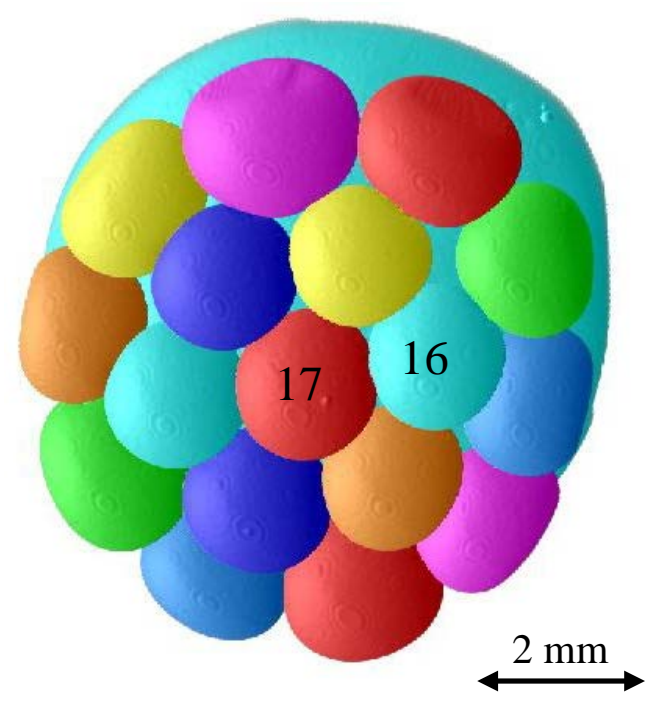

Figure 5. Bubbles displayed as in Fig. 3 but for a later stage: a) 16 min, b) 17 min. Bubble number 16 is displaced by the incoming number 17 . 


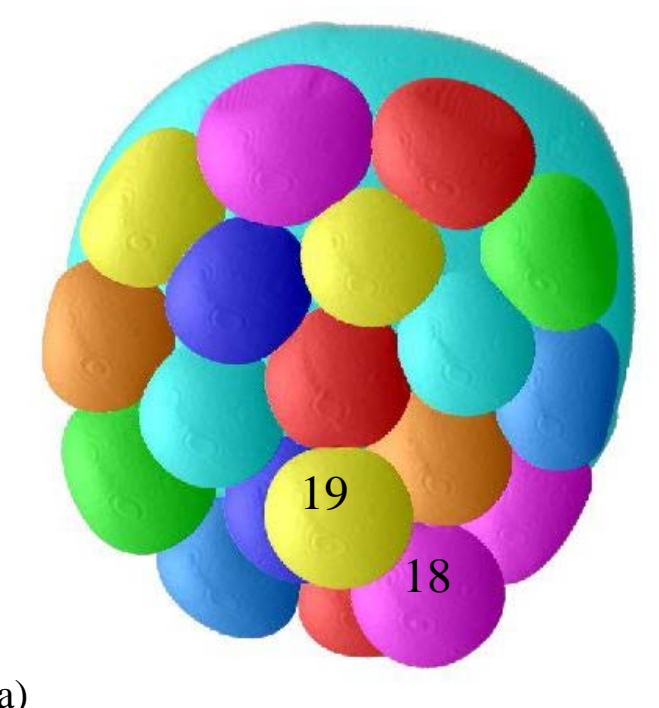

b)

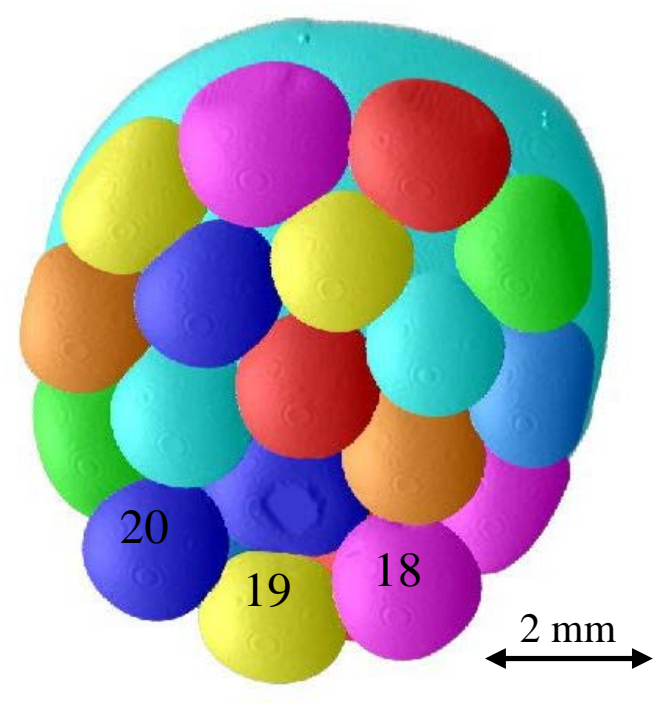

Figure 6. Bubbles displayed as in Fig. 3 but for a later stage during the build-up of the $2^{\text {nd }}$ layer (bubbles 18-19): a) 19 min., b) 20 min. Ejection of bubble number 19 by incoming number 20 is shown. 
a)

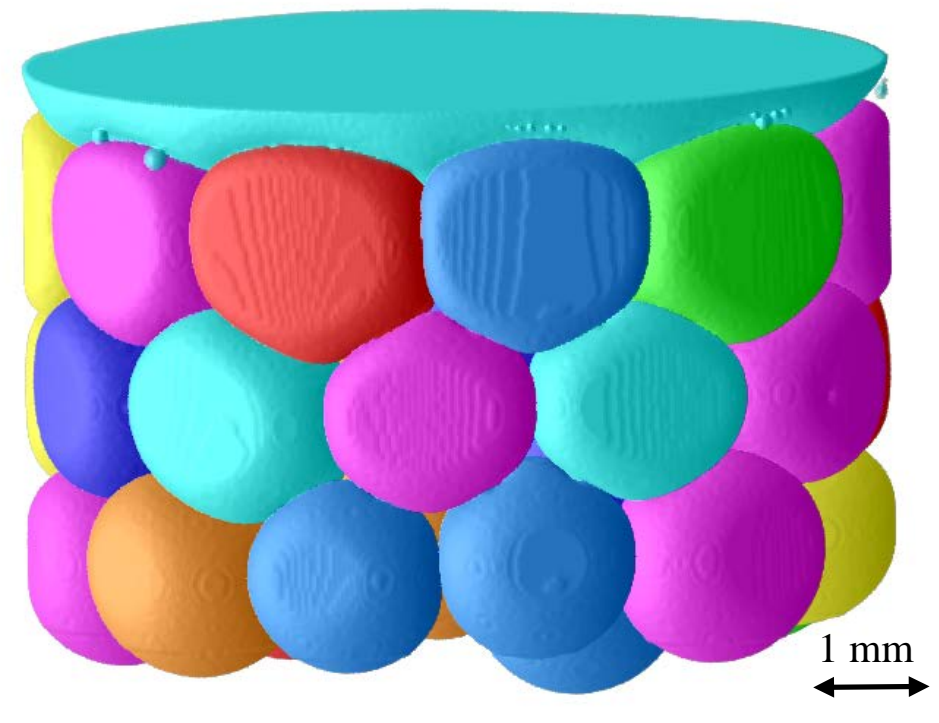

b)

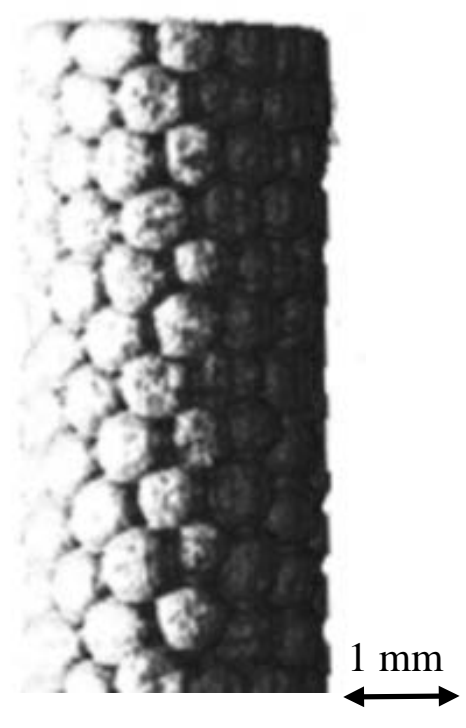

Figure 7. 3D rendering of tomography taken after all 48 bubbles had been deposited. It shows the arrangement of the bubbles situated next to the wall of the tube (outer ring) in all 3 layers produced. The direction of view is from the side other than in Figs. 3 to 6. b) A columnar foam made of microbubbles extracted from Ref [27] for matters of comparison. 


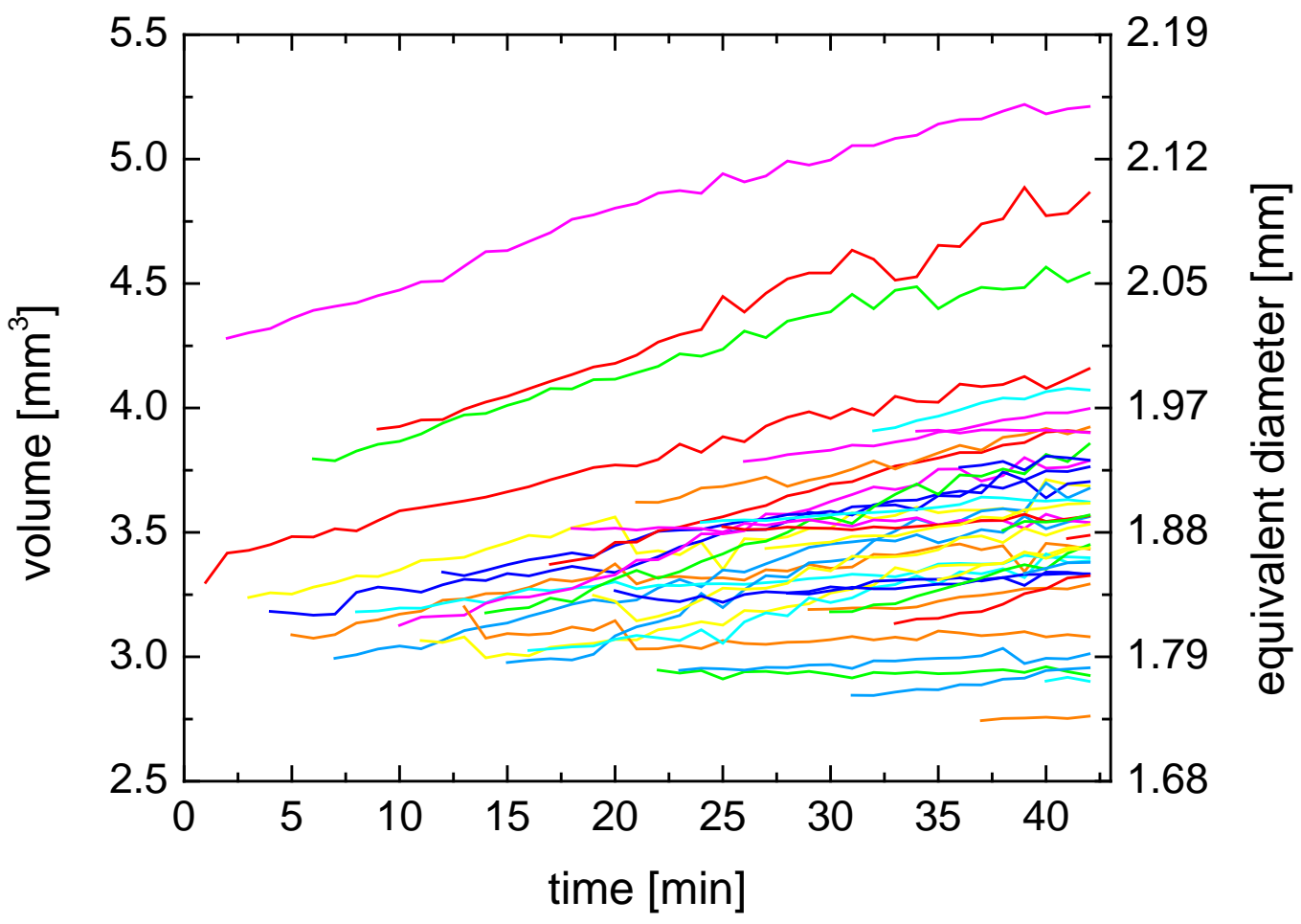

Figure 8. Evolution of bubble volume and equivalent diameter over the first $42 \mathrm{~min}$. for each individual bubble. 